\title{
Analysis of Present Status for Surgery of Benign Prostatic Hyperplasia in Korea Using Nationwide Healthcare System Data
}

\author{
Byeong Jo Jeon ${ }^{1}$, Hong Chung 2 , Jae Hyun Bae ${ }^{1}$, Han Jung ${ }^{3}$, Jeong Gu Lee ${ }^{1}$, Hoon Choi ${ }^{1}$ \\ ${ }^{1}$ Department of Urology, Korea University Medical Center, Korea University College of Medicine, Ansan, Korea \\ ${ }^{2}$ Department of Urology, Konkuk University Hospital, Konkuk University College of Medicine, Chungju, Korea \\ ${ }^{3}$ Department of Urology, Gachon University Gil Medical Center, Gachon University College of Medicine, Incheon, Korea
}

Purpose: There are no established statistical data available for the comparison of different surgical methods adopted for the resection of benign prostatic hyperplasia (BPH). This study investigates the present status related to BPH surgery in Korea for the past 8 years.

Methods: National-level data from the National Health Insurance Service and National Statistical Office were analyzed in this study. From 2010 to 2017, the trends of surgeries for BPH were reviewed according to the procedure code including transurethral resection of the prostate (TURP), holmium laser enucleation of the prostate (HoLEP), or high-power potassium titanyl phosphate (KTP), and this trend also analyzed by age, geographic distribution, and hospital type.

Results: Over the past 8 years, there was not much change in the total number of BPH-related surgeries (range, 10,39311,072). Although there was not much alteration in the number of conventional TURP (from 6,801 in 2010 to 6,645 in 2017), the number of HoLEP has dramatically increased (from 278 in 2010 to 3,805 in 2017). The number of HoLEP surgeries after 2011 exceeded the number of surgeries using KTP, and the gap is anticipated to rise. The number of surgeries by age group was most common in the 70s and the total number of surgeries is decreasing in all age groups; for HoLEP, the trend is steadily increasing over the age of 60 years. Most of the BPH surgeries were performed in metropolitan areas, such as Seoul, Gyeonggido, and Busan, and in larger hospitals compared to smaller hospital settings.

Conclusions: Through the data of the National Health Insurance Service, we could apprehend the present status of BPH-related surgery in Korea. Then, we could know about the trend according to several factors and we think these results will be valuable as academic references as well.

Keywords: Prostatic hyperplasia; Prostatectomy; Transurethral resection of prostate

- Grant/Fund Support: This research was supported by a grant of the Korean Urological Association.

- Research Ethics: This study was approved by the Institutional Review Board (IRB) of Korea University Medical Center (approval number: 2019AS0061). Due to the retrospective nature of this study, the IRB determined that we did not require informed consent documents from the patients. During the entire period of this study, patient information was anonymized or in de-identified status. We performed all procedures associated with the present study according to the Declaration of Helsinki guidelines.

- Conflict of Interest: No potential conflict of interest relevant to this article was reported.

\section{INTRODUCTION}

Benign prostatic hyperplasia (BPH) is a highly prevalent dis- ease in aging male patients, and it can negatively affect the quality of life (QoL) [1]. Korea has one of the most rapidly aging population in the world. BPH has been a major concern for
Corresponding author: Hoon Choi (iD https://orcid.org/0000-0002-8481-8103 Department of Urology, Korea University Ansan Hospital, Korea University College of Medicine, 123 Jeokgeum-ro, Danwon-gu, Ansan 15355, Korea E-mail: doc71377@naver.com / Tel: +82-31-412-5190 / Fax: +82-31-412-5194 Submitted: September 18, 2018 / Accepted after revision: January 1, 2019
This is an Open Access article distributed under the terms of the Creative Commons Attribution Non-Commercial License (http://creativecommons.org/licenses/by-nc/4.0/) which permits unrestricted non-commercial use, distribution, and reproduction in any medium, provided the original work is properly cited. 
both government and health care providers. According to data from the Health Insurance Review and Assessment (HIRA) agency in 2018, about 7,600,000 patients were diagnosed with BPH in 2010, which increased by approximately 1.2 million in 2017 [2]. This represents an annual increase of about 7\%, and a total increase of $58 \%$ from 2010. However, the actual number of patients with BPH was estimated to be much higher since not all the patients visit hospitals.

Treatment goals for patients with $\mathrm{BPH}$ are aimed at reducing symptoms, improving the QoL, and preventing disease progression $[1,3]$. In most cases, medication is the primary treatment modality [3]. Generally, the patients begin drug therapy in their 60s, and continue to take the drug for another 10 or 20 years considering the current life expectancy. Therefore, the time, cost, and treatment strategy should be well established. In fact, patients usually prefer noninvasive methods, and many efficacious drugs have been developed to reduce lower urinary tract symptoms caused by BPH. For a long time, transurethral resection of prostate (TURP) has been accepted as the standard treatment when the primary medical or surgical treatment fails, such as in cases of recurrent urinary tract infection, urinary retention, persistent prostatic bleeding or bladder stone [4]. Additionally, a number of safer and more effective procedures have been developed for $\mathrm{BPH}$ in the recent years, particularly the minimally invasive laser modalities including holmium laser enucleation of the prostate (HoLEP) and photoselective vaporization of the prostate (PVP), which have emerged as firstline surgical treatments to replace TURP. The surgical trends in BPH have been studied in several countries including United States, Canada, and Japan [5-7]. In a study to learn the surgical trend in Korea, Lee et al. [8] used HIRA's data to describe the nationwide incidence and treatment pattern of $\mathrm{BPH}$. However, this study was conducted between 2008 and 2011, and does not show any particular surgical trend for HoLEP, owing to its introduction only after 2010. Moreover, there are no other statistical studies available in Korea after this survey.

The purpose of this study was to analyze the frequency of surgical procedures and the overall surgical trends related to $\mathrm{BPH}$ surgery by region, hospital size, and year.

\section{MATERIALS AND METHODS}

With the approval of Institutional Review Board, we sorted the records of $\mathrm{BPH}$ patients in the HIRA database from the $\mathrm{Na}$ tional Health Insurance Service and National Statistical Office for analyzing the trends in $\mathrm{BPH}$-related surgeries during 8 years in Korea, from 2010 to 2017. Three of the most frequent procedures among the various surgeries, TURP, PVP using potassium titanyl phosphate (KTP), and HoLEP were analyzed. The diagnosis of BPH was defined in accordance with the 10th revision of International Classification of Diseases, as N 40.0 by primary or secondary diagnostic code. The patients were above 40 years of age, taking the prevalence of BPH into consideration. Exclusion criteria were patients with prostate malignancy, inflammatory disorders of the prostate, previous prostate operation, or neurologic problematic conditions that may influence voiding dysfunction. The condition of the procedure code was defined by the three surgical procedures described above: TURP (R3975), PVP using KTP (R3976) and HoLEP (R3977).

The number of operations (including detailed analysis of each surgical method) and the differences based on the type of hospital (clinic, hospital, general hospital, and superior general hospital) were recorded. The yearly changes of surgical incidence in each age group and the extent of surgery with the differences between the regions diagnosed with $\mathrm{BPH}$ and the regions undergoing surgery were also reviewed.

\section{RESULTS}

Our results were based on sample cohort big data analysis using the National Health Insurance database. Our cohort included patients diagnosed with BPH. Fig. 1 shows the total number of operations performed in the last 8 years. There was no significant difference in the total number of operations between 2010 $(10,393)$ and 2017 (11,072). TURP remained the most commonly performed operation for transurethral prostate surgery. Total number of conventional TURP remained stable (range, 5,732-6,944). KTP was the second common operation in 2010 and 2011, but the number of KTP showed a gradual decrease from 3,314 to 2,751, and eventually dropped to 622 in 2017. On the other hand, the number of HoLEP dramatically increased. In 2010, the number of HoLEP was only 278, but the number steadily increased, and finally exceeded the number of KTP in 2012, with a continuing steady increase in the difference.

Most surgeries for BPH are performed in large hospitals (superior general hospital or general hospital), which increased from $71 \%$ in 2010 to $81 \%$ in 2017 (Fig. 2). According to types of surgeries, about $80 \%$ cases of both TURP and HoLEP were performed in superior general hospital and general hospital in 2010 (5,580 and 221 cases) and 2017 (5,287 and 3,158 cases), 


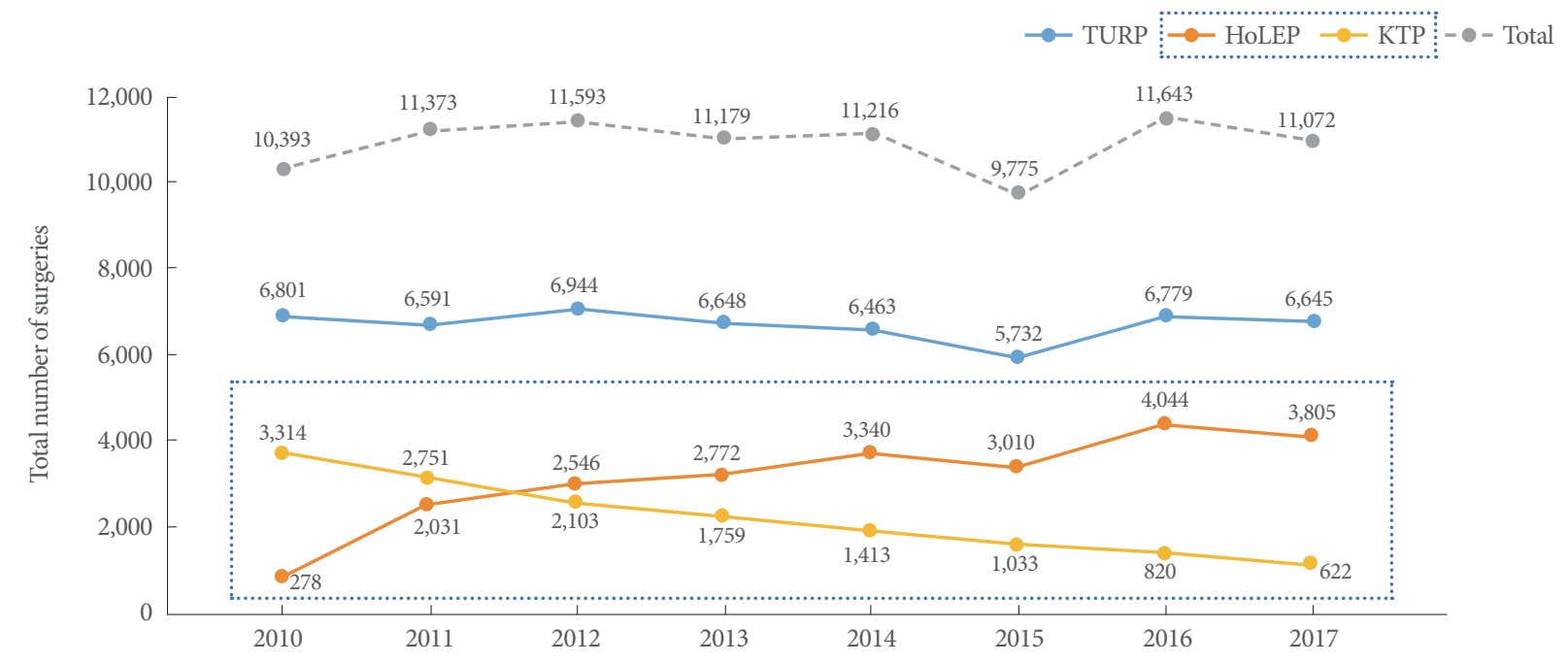

Fig. 1. Total number of operations for benign prostatic hyperplasia during the 8 years in Korea. TURP, transurethral resection of prostate; HoLEP, holmium enucleation of the prostate; KTP, high-power potassium titanyl phosphate.

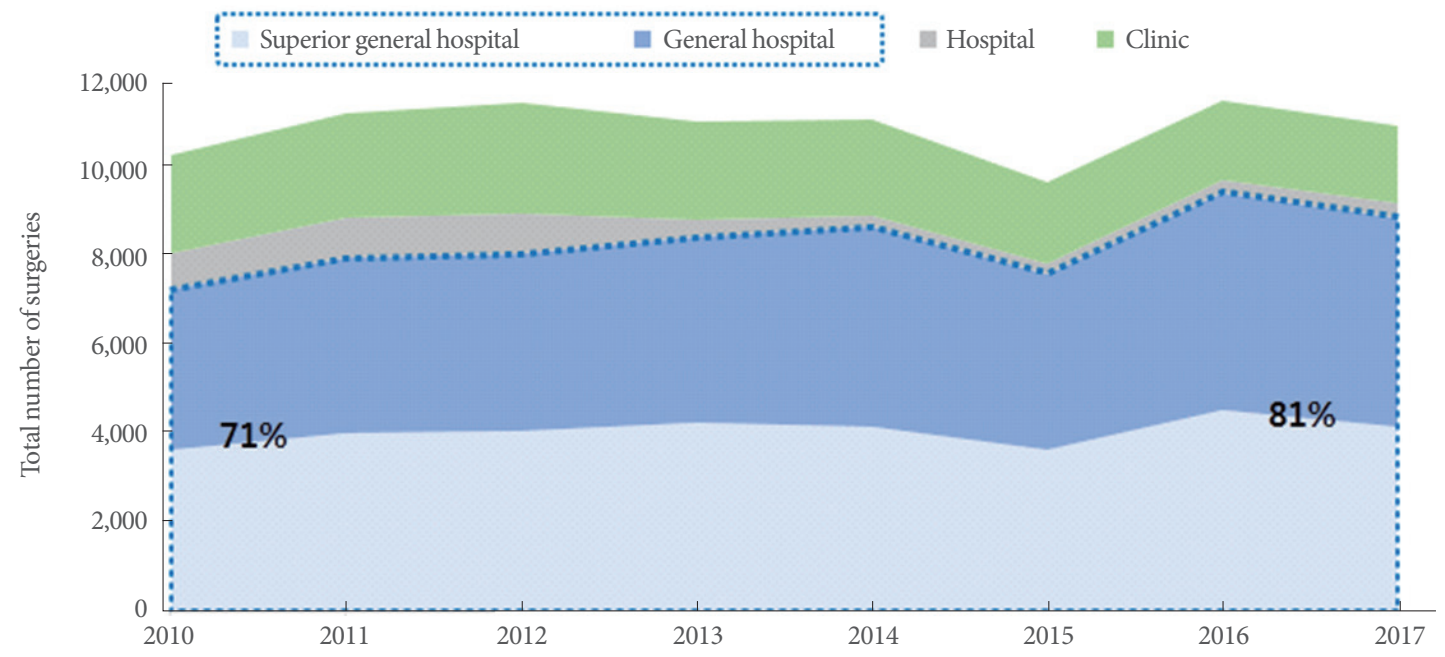

A

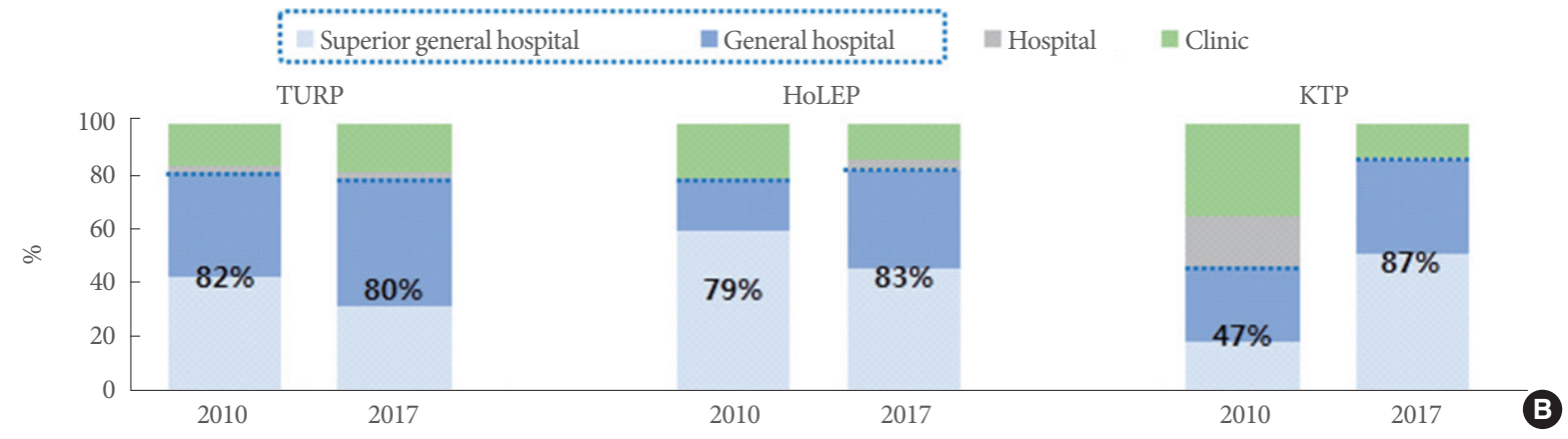

Fig. 2. The trend of surgery for benign prostatic hyperplasia (BPH) according to the type of hospital. (A) All surgeries. (B) TURP, HoLEP, and KTP. TURP, transurethral resection of prostate; HoLEP, holmium enucleation of the prostate; KTP, high-power potassium titanyl phosphate. 

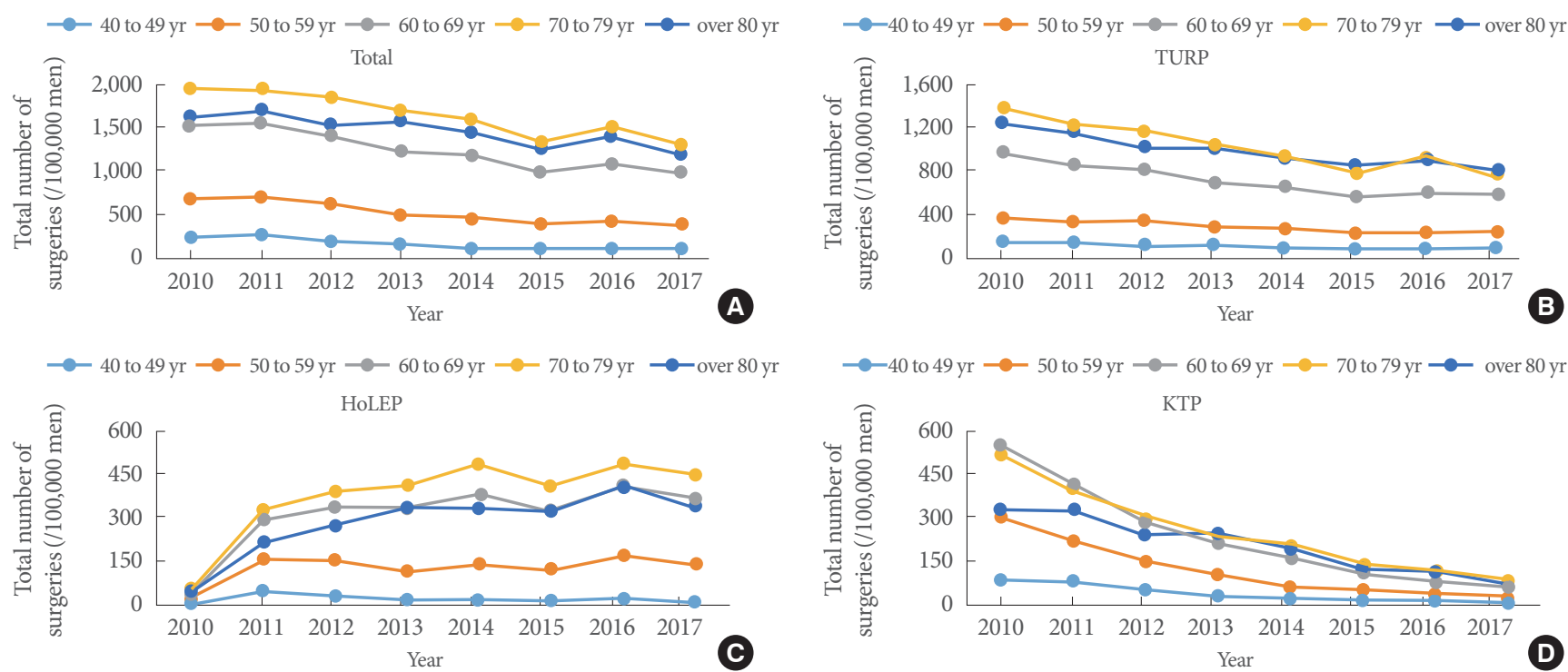

Fig. 3. The trend of age-related surgery for benign prostatic hyperplasia (/100,000 men). All surgeries (A), TURP (B), HoLEP (C), and KTP (D). TURP, transurethral resection of prostate; HoLEP, holmium enucleation of the prostate; KTP, high-power potassium titanyl phosphate.

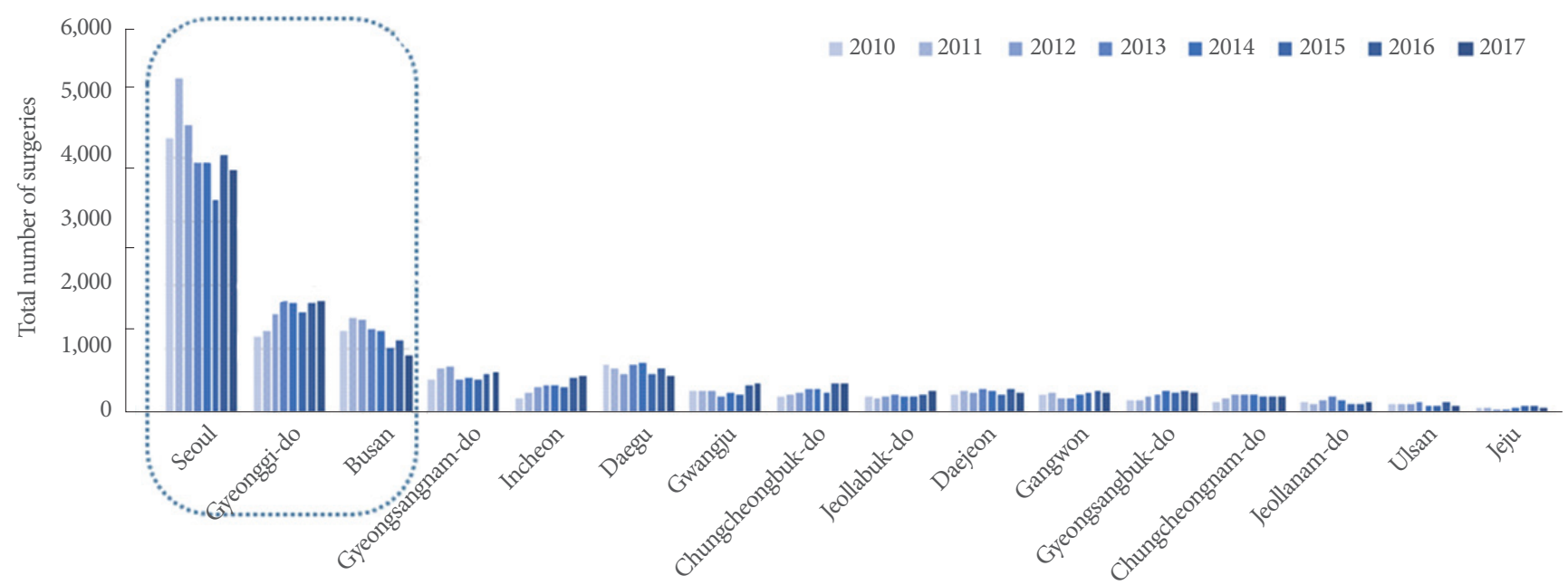

Fig. 4. The surgical trends according to the detailed regions of Korea.

respectively. Meanwhile $47 \%$ (1,543 cases) of KTP was performed in large hospitals and 35\% (1,146 cases) was performed in clinics in 2010. However, the proportion of large hospitals was increased to $87 \%$ (541 cases) and only 76 cases were performed at the clinics in 2017.

The trends in age-related surgery for $\mathrm{BPH}$ were analyzed in Fig. 3. Although the overall number of operations does not seem to be significantly different, the number of operations per 100,000 patients diagnosed with BPH tended to decrease in all age groups. KTP and TURP tended to decrease in all age groups, but HoLEP increased in age groups over 60 years. Since 2010, 70s were the most common age group undergoing surgeries, followed by the age group of 80s. The same trend was observed in the case of HoLEP as well, with the 70s age group being the most common age group, followed by the age group of $60 \mathrm{~s}$. However, in the TURP group, the age group of $80 \mathrm{~s}$ was undergoing more surgeries than the age group of 70 s since 2015.

Fig. 4 shows the surgical trends according to the detailed regions of Korea. The most common sites for surgery of $\mathrm{BPH}$ 
were Seoul, Gyeonggi-do, and Busan. The rate of surgery in these 3 areas accounted for $60 \%$ of all operations. Compared to other regions, it was found that many cases were performed uniquely in Seoul (range, 3,335-5,248), followed by Gyeonggido (range, 1,185-1,753), where the number of operations was seen to be steadily increasing in the recent years. A large number of surgeries were also performed in Busan, the second largest city in Korea after Seoul (range, 909-1,493).

\section{DISCUSSION}

$\mathrm{BPH}$ is a common disease of the elderly that may adversely affect the QoL. According to Lee et al. [8], 386,873 surgeries were identified with BPH in 2008. This corresponds to 2105 surgeries per 100,000 men in Korea. BPH can cause a lot of social costs, so treatment at an appropriate point in time is important. For a long time, TURP has been considered as the primary surgical treatment for $\mathrm{BPH}$ [4]. Recently, minimally invasive treatment methods using laser have been introduced. Currently available surgeries for BPH in Korea are TURP, PVP (KTP), HoLEP, open prostatectomy, transurethral needle ablation, and Prostate ligation (UroLift System, NeoTract, Inc., Pleasanton, CA, USA). All of the procedures mentioned in this article are available in Korea. HoLEP is rapidly replacing other open BPH operations since its introduction, and open prostatectomy is no longer performed. Currently, the most commonly performed surgeries in Korea are TURP, KTP, and HoLEP. The health insurance claim data provided by HIRA is a representative data of the medical contents of the all citizens and has representative and inclusiveness. Unlike primary data collected under strict control, claim data reflects the real society where researchers can conduct research based on observation of trends that consider the actual health care environment rather than the limited experimental environment. So, the results seem to be representative of the actual clinical scenarios in Korea.

In our present study, the traditional TURP was found to be the most common among the prostate surgeries, and the number of surgeries remained stable for 8 years. The number of HoLEP has surged rapidly, surpassing the number of KTPs since 2012. These results were comparable to other studies conducted in other countries including USA, Canada, and Japan [5-7]. Previous studies have reported TURP to be the most common procedure without a significant difference in the number of operations. Further, laser treatments including HoLEP and PVP showed an increase forming the second most common treat- ment for BPH. However, the rates of total number of operations with TURP and laser therapies vary between countries. In the USA, laser therapies account for 50\% of TURP surgeries, and in Canada, TURP accounts for more than $90 \%$ of all procedures while laser therapies account for only 7.6\%. These differences are due to the differences in the study period, the time of introduction of BPH procedures, acquisition costs, different reimbursement incentives, and insurance policies. In addition, BPH surgeries are mainly conducted in the big cities or metropolitan areas where large hospitals are located rather than based on the absolute number or regional distribution of urology patients. This in turn implies that most of the HoLEP surgeries are mainly performed in large hospitals. In addition, the number of operations performed in local clinics have decreased, with an increase in the number of surgeries performed in general hospitals since 2012, which has also contributed to the increased number of HoLEP. This trend can also be seen in the Canadian BPH surgeries [6]. The total number of TURPs and the proportion of laser procedures in the total number of surgeries were higher in big cities. In this study, the number of operations per 100,000 $\mathrm{BPH}$ patients tended to decrease in all age groups. This decrease might be attributed to the large number of new drugs that have been recently developed for the lower urinary tract symptoms due to $\mathrm{BPH}$. It has been reported that the most common age group to receive the $\mathrm{BPH}$ surgeries is the 70s. This might indicate that the overall health status and morbidity of the BPH surgeries have improved in the older age groups.

TURP has been the gold standard for surgical treatment for BPH for a long time due to its effectiveness and low cost [9]. TURP was reported to be the most commonly performed BPH-related surgeries in Korea [8]. TURP using resectoscope is still the most familiar method for many urologists. In this study, patients in the 60s constitute the second most common age group in HoLEP and in TURP, with 80 s being the second most frequently operated group of patients. This could be because the relatively younger and healthier patients were more likely to receive new procedures like laser therapies, whereas the elderly patients with greater comorbidities tend to hesitate to undergo newer and less established therapies [7]. However, TURP has some potential risks, such as postoperative bleeding, clot retention, prolonged catheterization times, long hospital stays and transurethral resection syndrome [10]. In recent years, laser therapies including KTP or HoLEP, have been shown to produce comparable or superior surgical outcomes than TURP and are preferred by many surgeons due to fewer 
postoperative complications.

KTP was spotlighted in the early days due to the minimal learning curve for the vaporization technique [11]. KTP is basically similar to TURP and can therefore be easily performed by urologists who have experience in TURP surgery. Further, even surgeons with less experience with KTP were able to provide significant relief of symptoms and improvement in urodynamic parameters with the safe and effective removal of prostatic tissue. For this reason, KTP was mainly performed at the local clinics in the early stage. But recently, it has been steadily decreasing due to some limitations of KTP. Recently, there have been reports on the efficacy and safety of KTP for prostates of more than $150 \mathrm{~mL}$ [12]. However, there are limited studies on the efficacy of KTP on large prostate, although the short- and medium-term data for small and medium prostates are well established. Horasanli et al. [13] reported that KTP showed worse outcomes than TURP for large prostatic gland $(>70 \mathrm{~mL})$ on postoperative International Prostate Symptom Score (IPSS)/ QoL, maximal flow rate (Qmax), and postvoided urine volume (PVR). According to Elmansy et al. [14], KTP requires multiple laser fibers for completion of surgery and a higher energy setting than HoLEP. Further, 22\% of patients undergoing KTP switched to HoLEP or TURP. In these cases, impaired vision from bleeding could not be controlled with the KTP laser.

Many studies report that HoLEP is as effective or better than other surgical options [14-16]. Gilling et al. [16] reported that HoLEP showed an increased amount of tissue removal, shorter catheterization time, shorter hospital length of stay, and lower transfusion rates than TURP. Additionally, there was no reoperation for $\mathrm{BPH}$ in the patients who underwent HoLEP compared to $18 \%$ reoperation rate in patients who underwent TURP. In the randomized controlled trial comparing HoLEP with KTP, there was no significant difference in the IPSS, QoL, or sexual function between the 2 groups, except for the high postoperative Qmax and the lower PVR in the HoLEP group [14]. Contrary to TURP, HoLEP can replace the open prostatectomy as it is a size-independent procedure, and TUR syndrome has never been reported even with large prostate $[17,18]$. According to Kuntz el al. [18], HoLEP has been shown to have similar efficacy as open prostatectomy in resecting large prostatic adenoma greater than $100 \mathrm{~g}$ with radically decreased hospital length of stay, catheterization times, blood loss, and transfusion rates. Nevertheless, HoLEP has not yet replaced TURP. A major limitation in popularizing HoLEP is thought to be its technical difficulty and steep learning curve of the procedure.
According to a recent analysis, 50-60 cases were required to effectively perform enucleation. Efficient morcellation with stabilization was reported after 60 cases [19]. In addition to this relatively high learning curve, a high price of the generator and morcellator also serves as an obstacle to the popularity of HoLEP. However, in the end, HoLEP may be able to overtake TURP in a few years, due to its similar efficacy to procedures such as TURP, open prostatectomy, and other laser therapies and fewer complications, shorter catheterization time, shorter hospital stays, and decreased blood loss.

There are some limitations in this study. Firstly, the data in this study did not include various clinical information associated with BPH such as IPSS, serum PSA level, and prostate size. In addition, $\mathrm{BPH}$ medication history such as use of alphablocker and 5-alpha reductase inhibitor (5-ARI) is not included. Therefore, the various factors involved in considering surgery or surgical methods could not be analyzed. Unmeasured factors such as physician's proficiency and preference, which may vary depending on the procedure type, that may affect the choice of the surgical procedure could not be explained. Secondly, this study analyzed the number of operations using the code provided by HIRA, therefore the number of surgeries performed do not represent the number of patients. Hence, this data cannot be distinguished even if reoperation is performed. However, in the case of HoLEP, the reoperation rate was reported as $1 \%$ within 5 to 10 years [20], together with an average reoperation rate of $7.4 \%$ and $5 \%-6 \%$ for TURP and PVP, respectively $[17,21]$. Therefore, there is no problem in the representation of data used in this study. In addition, the Urolift has recently been introduced in Korea and is currently being practiced at local clinics. It is not yet popular because health insurance has not yet been applied. Although Urolift is currently performed in limited outpatients with high comorbidities due to lack of long-term outcome, it can be applied to more patients since it is an easy and safe procedure with high accessibility and lower incidence of adverse effects [22,23]. It is expected that a significant contribution will be made to one of the alternatives for surgical treatment of BPH.

Despite these limitations, this study is meaningful as it is conducted on the whole people based on nationwide data. To the best of our knowledge, this is the first study to report on the surgical trend of BPH procedures within the last 8 years in Korea. Furthermore, the trends in this study are similar to surgical trends of $\mathrm{BPH}$ in other countries [5-7]. Surgical procedures of $\mathrm{BPH}$ have been increasing for the last 8 years in Korea and 
these results seem to be representative of the actual clinical condition. Although many new drugs including alpha-blocker and 5-ARI have recently been developed for the lower urinary tract symptoms due to $\mathrm{BPH}$, more number of elderly patients are expected to undergo surgery regardless of the surgical methods for $\mathrm{BPH}$ with the increasing elderly population and improvements in the overall health levels.

In conclusion, in Korea, during the last 8 years, TURP was the most commonly performed $\mathrm{BPH}$ procedure, followed by HoLEP and KTP. Since 2011, HoLEP has continued to increase and KTP has continued to decline. The most common age group to receive $\mathrm{BPH}$ surgeries is over 70 years old. Additionally, BPH surgeries have been carried out mainly in big cities with large hospitals.

\section{AUTHOR CONTRIBUTION STATEMENT}

- Full access to all the data in the study and takes responsibility for the integrity of the data and the accuracy of the data analysis: $H C$

- Study concept and design: JGL, HC

- Acquisition of data: $H J, H C, H C, B J J$

- Analysis and interpretation of data: $H C, B J J$

- Drafting of the manuscript: $H C, B J J$

- Critical revision of the manuscript for important intellectual content: $J H B, H C$

-Statistical analysis: $H C, B J J$

- Administrative, technical, or material support: $H C$

- Study supervision: JGL, HC

\section{REFERENCES}

1. McConnell JD, Roehrborn CG, Bautista OM, Andriole GL Jr, Dixon CM, Kusek JW, et al. The long-term effect of doxazosin, finasteride, and combination therapy on the clinical progression of benign prostatic hyperplasia. N Engl J Med 2003;349:2387-98.

2. Health Insurance Review \& Assessment Service [Internet]. Wonju (Korea): Health Insurance Review \& Assessment Service; c2015 [updated 2018 Sep 14; cited 2018 Sep 26]. Available from: http:// opendata.hira.or.kr/op/opc/olapDiagBhvInfo.do.

3. Auffenberg GB, Helfand BT, McVary KT. Established medical therapy for benign prostatic hyperplasia. Urol Clin North Am 2009;36:443-59, v-vi.

4. Nickel JC, Méndez-Probst CE, Whelan TF, Paterson RF, Razvi H. 2010 Update: guidelines for the management of benign prostatic hyperplasia. Can Urol Assoc J 2010;4:310-6.

5. Masumori N, Kamoto T, Seki N, Homma Y; Committee for Clinical Guideline for Benign Prostatic Hyperplasia. Surgical procedures for benign prostatic hyperplasia: a nationwide survey in Japan. Int $J$ Urol 2011;18:166-70.

6. Hueber PA, Zorn KC. Canadian trend in surgical management of benign prostatic hyperplasia and laser therapy from 2007-2008 to 2011-2012. Can Urol Assoc J 2013;7:E582-6.

7. Schroeck FR, Hollingsworth JM, Kaufman SR, Hollenbeck BK, Wei JT. Population based trends in the surgical treatment of benign prostatic hyperplasia. J Urol 2012;188:1837-41.

8. Lee YJ, Lee JW, Park J, Seo SI, Chung JI, Yoo TK, et al. Nationwide incidence and treatment pattern of benign prostatic hyperplasia in Korea. Investig Clin Urol 2016;57:424-30.

9. Gratzke C, Bachmann A, Descazeaud A, Drake MJ, Madersbacher $S$, Mamoulakis $C$, et al. EAU guidelines on the assessment of nonneurogenic male lower urinary tract symptoms including benign prostatic obstruction. Eur Urol 2015;67:1099-109.

10. Rassweiler J, Teber D, Kuntz R, Hofmann R. Complications of transurethral resection of the prostate (TURP)--incidence, management, and prevention. Eur Urol 2006;50:969-79.

11. Seki N, Nomura H, Yamaguchi A, Naito S. Evaluation of the learning curve for photoselective vaporization of the prostate over the course of 74 cases. J Endourol 2008;22:1731-5.

12. Stone BV, Chughtai B, Forde JC, Tam AW, Lewicki P, Te AE. Safety and efficacy of GreenLight XPS laser vapoenucleation in prostates measuring over 150mL. J Endourol 2016;30:906-12.

13. Horasanli K, Silay MS, Altay B, Tanriverdi O, Sarica K, Miroglu C. Photoselective potassium titanyl phosphate (KTP) laser vaporization versus transurethral resection of the prostate for prostates larger than $70 \mathrm{~mL}$ : a short-term prospective randomized trial. Urology 2008;71:247-51.

14. Elmansy H, Baazeem A, Kotb A, Badawy H, Riad E, Emran A, et al. Holmium laser enucleation versus photoselective vaporization for prostatic adenoma greater than $60 \mathrm{ml}$ : preliminary results of a prospective, randomized clinical trial. J Urol 2012;188:216-21.

15. Montorsi F, Naspro R, Salonia A, Suardi N, Briganti A, Zanoni M, et al. Holmium laser enucleation versus transurethral resection of the prostate: results from a 2-center, prospective, randomized trial in patients with obstructive benign prostatic hyperplasia. J Urol 2004;172(5 Pt 1):1926-9.

16. Gilling PJ, Wilson LC, King CJ, Westenberg AM, Frampton CM, Fraundorfer MR. Long-term results of a randomized trial comparing holmium laser enucleation of the prostate and transurethral resection of the prostate: results at 7 years. BJU Int 2012;109:408-11. 
17. Ahyai SA, Gilling P, Kaplan SA, Kuntz RM, Madersbacher S, Montorsi $\mathrm{F}$, et al. Meta-analysis of functional outcomes and complications following transurethral procedures for lower urinary tract symptoms resulting from benign prostatic enlargement. Eur Urol 2010;58:384-97.

18. Kuntz RM, Lehrich K, Ahyai SA. Holmium laser enucleation of the prostate versus open prostatectomy for prostates greater than 100 grams: 5-year follow-up results of a randomised clinical trial. Eur Urol 2008;53:160-6.

19. Brunckhorst O, Ahmed K, Nehikhare O, Marra G, Challacombe B, Popert R. Evaluation of the learning curve for holmium laser enucleation of the prostate using multiple outcome measures. Urology 2015;86:824-9.

20. Elmansy HM, Kotb A, Elhilali MM. Holmium laser enucleation of the prostate: long-term durability of clinical outcomes and complication rates during 10 years of followup. J Urol 2011;186:1972-6.

21. Madersbacher S, Lackner J, Brössner C, Röhlich M, Stancik I, Willinger $\mathrm{M}$, et al. Reoperation, myocardial infarction and mortality after transurethral and open prostatectomy: a nation-wide, longterm analysis of 23,123 cases. Eur Urol 2005;47:499-504.

22. Bozkurt A, Karabakan M, Keskin E, Hirik E, Balci MB, Nuhoglu B. Prostatic urethral lift: a new minimally invasive treatment for lower urinary tract symptoms secondary to benign prostatic hyperplasia. Urol Int 2016;96:202-6.

23. Nunes RLV, Antunes AA, Constantin DS. Contemporary surgical treatment of benign prostatic hyperplasia. Rev Assoc Med Bras (1992) 2017;63:711-6. 